\title{
THE INTEGRAL WAVELET TRANSFORM IN WEIGHTED SOBOLEV SPACES
}

\author{
NGUYEN MINH CHUONG AND TA NGOC TRI
}

Received 27 September 2001

The integral wavelet transform is defined in weighted Sobolev spaces, in which some properties of the transform as well as its asymptotical behaviour for small dilation parameter are studied.

\section{Preliminaries and notations}

It is well known that the integral wavelet transform is a very powerful tool to study sciences and technology. In [5] wavelet theory has been investigated in very much functional spaces, even in BMO, VMO (for further details of those spaces, please refer to [5] and the references therein) even for pseudodifferential operators, however, the integral wavelet transform in weighted Sobolev spaces has not been studied yet neither in [5] nor in any other work.

The aim of this paper is to study this unsolved problem.

Let $\omega_{\mu}(x) \in L^{\infty}\left(\mathbb{R}^{n}\right), \omega_{\mu}(x)>0$, for almost all $x \in \mathbb{R}^{n}$ and for each $x$,

$$
\omega_{\mu}(x+y) \leq C_{1, \mu} \omega_{\mu}(x)
$$

for almost all $y \in \mathbb{R}^{n}$, where $\mu$ is a multi-index.

We use the Sobolev space with weighted norm defined as follows:

$$
W_{\omega}^{m, p}\left(\mathbb{R}^{n}\right)=\left\{f \in L^{p}\left(\mathbb{R}^{n}\right)\left|\partial^{k} f \in L^{p}\left(\mathbb{R}^{n}\right),\right| k \mid \leq m\right\}
$$

equipped with the norm

$$
\|f\|_{m, p, \omega}=\sum_{|\mu| \leq m}\left(\int_{\mathbb{R}^{n}} \omega_{\mu}(x)\left|\partial^{\mu} f(x)\right|^{p} d x\right)^{1 / p}<\infty
$$

where $\mu=\left(\mu_{1}, \ldots, \mu_{n}\right),|\mu|=\mu_{1}+\cdots+\mu_{n}, \mu_{i} \geq 0$.

Copyright (C) 2002 Hindawi Publishing Corporation Abstract and Applied Analysis 7:3 (2002) 135-142

2000 Mathematics Subject Classification: 42Cxx, 42C40, 65Txx, $65 T 60$

URL: http://dx.doi.org/10.1155/S1085337502000775 
136 The integral wavelet transform in weighted Sobolev spaces

Let $\mathscr{Y}\left(\mathbb{R}^{n}\right)$ be the Schwartz space of all differentiable functions $\varphi$ on $\mathbb{R}^{n}$ such that for all multi-indices $\alpha$ and $\beta$

$$
\sup _{x \in \mathbb{R}^{n}}\left|x^{\alpha}\left(D^{\beta} \varphi(x)\right)\right|<\infty .
$$

The Fourier transform $\mathscr{F}: f \mapsto \hat{f}$ is given by

$$
\hat{f}(y)=(2 \pi)^{-n / 2} \int_{\mathbb{R}^{n}} f(x) e^{-i(x, y)} d x,
$$

where $(x, y)=x_{1} y_{1}+\cdots+x_{n} y_{n}, x=\left(x_{1}, \ldots, x_{n}\right), y=\left(y_{1}, \ldots, y_{n}\right)($ see $[1,2,4])$.

As traditionally, it is not difficult to prove that $\mathscr{S}\left(\mathbb{R}^{n}\right)$ is dense in $W_{\omega}^{m, p}\left(\mathbb{R}^{n}\right)$. Now we recall that a basic wavelet is a nontrivial function $\psi \in L^{1}\left(\mathbb{R}^{n}\right)$ such that its integral on $\mathbb{R}^{n}$ is 0 and its Fourier transform $\hat{\psi}(\xi)$ satisfies the condition

$$
(2 \pi)^{n} \int_{0}^{\infty} \frac{|\hat{\psi}(a \xi)|^{2}}{a} d a,
$$

denoted by $C_{\psi}$ which is a constant for every $\xi \neq 0$ and $C_{\psi} \neq 0$.

With a basic wavelet $\psi$ and a function $f \in \mathscr{S}\left(\mathbb{R}^{n}\right)$, we define the following integral:

$$
\left(L_{\psi} f\right)(b, a)=\frac{1}{\sqrt[2 n]{C_{\psi}}} \frac{1}{\sqrt{|a|^{n}}} \int_{\mathbb{R}^{n}} \bar{\psi}\left(\frac{t-b}{a}\right) f(t) d t
$$

where $b \in \mathbb{R}^{n}$ and $a \in \mathbb{R} \backslash\{0\}$ (see $\left.[3,5,6]\right)$.

\section{Some properties}

Proposition 2.1. If $f \in \mathscr{Y}\left(\mathbb{R}^{n}\right)$ then

$$
\left\|\left(L_{\psi} f\right)(\cdot, a)\right\|_{m, p, \omega} \leq C\|f\|_{m, p, \omega}
$$

where $a \in \mathbb{R}, a \neq 0$ and fixed, $C$ is a constant independent of $f$.

Proof. Obviously we have

$$
\left(L_{\psi} f\right)(\cdot, a)=\frac{1}{\sqrt[2 n]{C_{\psi}}}\left(f * D^{-a} \bar{\psi}\right)(\cdot)
$$

where $D^{a}: L^{2}\left(\mathbb{R}^{n}\right) \rightarrow L^{2}\left(\mathbb{R}^{n}\right)$ and $\left(D^{a} \psi\right)(a)=|a|^{-n / 2} \psi(x / a) ; a \neq 0$.

Since $f \in \mathscr{Y}\left(\mathbb{R}^{n}\right)$ the differentiation and integration can be interchanged

$$
\partial^{\mu}\left(L_{\psi} f\right)(\cdot, a)=\frac{1}{\sqrt[2 n]{C_{\psi}}}\left(D^{-a} \bar{\psi} * \partial^{\mu} f\right)(\cdot) .
$$


It is not difficult to see that

$$
\begin{aligned}
& \left\|\left(L_{\psi} f\right)(\cdot, a)\right\|_{m, p, \omega} \\
& \quad=\sum_{|\mu| \leq m}\left(\int_{\mathbb{R}^{n}} \omega_{\mu}(\cdot)\left|\partial^{\mu}\left(L_{\psi} f\right)(\cdot, a)\right|^{p} d(\cdot)\right)^{1 / p} \\
& \quad=\sum_{|\mu| \leq m} \frac{1}{\sqrt[2 n]{C_{\psi}}}\left(\int_{\mathbb{R}^{n}} \omega_{\mu}(x)\left|\int_{\mathbb{R}^{n}}\left(\partial^{\mu} f\right)(x-y)\left(D^{-a} \bar{\psi}\right)(y) d y\right|^{p} d x\right)^{1 / p} \\
& \quad \leq \frac{1}{\sqrt[2 n]{C_{\psi}}} \sum_{|\mu| \leq m} \int_{\mathbb{R}^{n}}\left(\int_{\mathbb{R}^{n}}\left|\left(\partial^{\mu} f\right)(x-y)\right|^{p}\left|\left(D^{-a} \bar{\psi}\right)(y)\right|^{p} \omega_{\mu}(x) d x\right)^{1 / p} d y \\
& \quad \leq \frac{1}{\sqrt[2 n]{C_{\psi}}} \sum_{|\mu| \leq m} \int_{\mathbb{R}^{n}}\left|D^{-a} \bar{\psi}(y)\right|\left(\int_{\mathbb{R}^{n}} \omega_{\mu}(x)\left|\left(\partial^{\mu} f\right)(x-y)\right|^{p} d x\right)^{1 / p} d y .
\end{aligned}
$$

However,

$$
\begin{aligned}
\int_{\mathbb{R}^{n}} \omega_{\mu}(x)\left|\left(\partial^{\mu} f\right)(x-y)\right|^{p} d x & =\int_{\mathbb{R}^{n}} \omega_{\mu}(u+y)\left|\left(\partial^{\mu} f\right)(u)\right|^{p} d u \\
& \leq C_{1, \mu} \int_{\mathbb{R}^{n}} \omega_{\mu}(u)\left|\left(\partial^{\mu} f\right)(u)\right|^{p} d u .
\end{aligned}
$$

Consequently,

$$
\begin{aligned}
& \left(\int_{\mathbb{R}^{n}} \omega_{\mu}(\cdot)\left|\partial^{\mu}\left(L_{\psi} f\right)(\cdot, a)\right|^{p} d(\cdot)\right)^{1 / p} \\
& \quad \leq \frac{\left(C_{1, \mu}\right)^{1 / p}}{\sqrt[2 n]{C_{\psi}}} \int_{\mathbb{R}^{n}}\left|D^{-a} \bar{\psi}(y)\right| d y\left(\int_{\mathbb{R}^{n}} \omega_{\mu}(x)\left|\left(\partial^{\mu} f\right)(x)\right|^{p} d x\right)^{1 / p} \\
& \quad \leq C_{\mu}\left(\int_{\mathbb{R}^{n}} \omega_{\mu}(x)\left|\left(\partial^{\mu} f\right)(x)\right|^{p} d x\right)^{1 / p},
\end{aligned}
$$

where

$$
C_{\mu}=\frac{\left(C_{1, \mu}\right)^{1 / p}}{\sqrt[2 n]{C_{\psi}}}|a|^{n / 2}\|\psi\|_{1}
$$

Therefore,

$$
\left\|\left(L_{\psi} f\right)(\cdot, a)\right\|_{m, p, \omega} \leq C \sum_{|\mu| \leq m}\left(\int_{\mathbb{R}^{n}} \omega_{\mu}(x)\left|\left(\partial^{\mu} f\right)(x)\right|^{p} d x\right)^{1 / p},
$$

where

$$
C=\max _{|\mu| \leq m} C_{\mu}
$$


138 The integral wavelet transform in weighted Sobolev spaces

that is,

$$
\left\|\left(L_{\psi} f\right)(\cdot, a)\right\|_{m, p, \omega} \leq C\|f\|_{m, p, \omega} .
$$

By Proposition 2.1, we extend $\left(L_{\psi} f(\cdot, a)\right)$ for fixed $a$ to a continuous mapping from $W_{\omega}^{m, p}\left(\mathbb{R}^{n}\right)$ to itself. It is called the integral wavelet transform in weighted Sobolev space.

THEOREM 2.2. If $\psi$ and $\varphi$ are basic wavelets and $f, g$ belong to $W_{\omega}^{m, p}\left(\mathbb{R}^{n}\right)$, then the following estimate holds true:

$$
\begin{aligned}
& \left\|\left(L_{\psi} f\right)(\cdot, a)-\left(L_{\varphi} g\right)(\cdot, a)\right\|_{m, p, \omega} \\
& \quad \leq C^{1}|a|^{n / 2}\left(\left\|\frac{\psi}{\sqrt[2 n]{C_{\psi}}}-\frac{\varphi}{\sqrt[2 n]{C_{\varphi}}}\right\|_{1}\|f\|_{m, p, \omega}+\frac{\|\varphi\|_{1}}{\left.\sqrt[2 n]{C_{\varphi}}\|f-g\|_{m, p, \omega}\right),}\right.
\end{aligned}
$$

where $C^{1}$ is a constant independent of $f$ and $g$.

Proof. It is sufficient to prove the case $f, g \in \mathscr{Y}\left(\mathbb{R}^{n}\right)$.

Obviously

$$
\begin{aligned}
& \left(\int_{\mathbb{R}^{n}} \omega_{\mu}(\cdot)\left|\partial^{\mu}\left[L_{\psi} f-L_{\varphi} g\right](\cdot, a)\right|^{p} d(\cdot)\right)^{1 / p} \\
& \quad=\left\{\int_{\mathbb{R}^{n}} \omega_{\mu}(\cdot)\left|\partial^{\mu} f *\left(\frac{D^{-a} \bar{\psi}}{\sqrt[2 n]{C_{\psi}}}-\frac{D^{-a} \bar{\varphi}}{\sqrt[2 n]{C_{\varphi}}}\right)(\cdot)\right|^{p} d(\cdot)\right\}^{1 / p} \\
& \quad=\left\{\int_{\mathbb{R}^{n}} \omega_{\mu}(x)\left|\int_{\mathbb{R}^{n}}\left(\partial^{\mu} f\right)(x-y)\left(\frac{D^{-a} \bar{\psi}}{\sqrt[2 n]{C_{\psi}}}-\frac{D^{-a} \bar{\varphi}}{\sqrt[2 n]{C_{\varphi}}}\right)(y) d y\right|^{p} d x\right\}^{1 / p} \\
& \quad \leq \int_{\mathbb{R}^{n}}\left|\left(\frac{D^{-a} \bar{\psi}}{\sqrt[2 n]{C_{\psi}}}-\frac{D^{-a} \bar{\varphi}}{\sqrt[2 n]{C_{\varphi}}}\right)(y)\right|\left(\int_{\mathbb{R}^{n}} \omega_{\mu}(x)\left|\left(\partial^{\mu} f\right)(x-y)\right|^{p} d x\right)^{1 / p} d y \\
& \quad \leq C_{2, \mu}\left(\int_{\mathbb{R}^{n}} \omega_{\mu}(x)\left|\left(\partial^{\mu} f\right)(x)\right|^{p} d x\right)^{1 / p},
\end{aligned}
$$

where

$$
C_{2, \mu}=|a|^{n / 2}\left\|\frac{\psi}{\sqrt[2 n]{C_{\psi}}}-\frac{\varphi}{\sqrt[2 n]{C_{\varphi}}}\right\|_{1}\left(C_{1, \mu}\right)^{1 / p}
$$


So

$$
\left\|\left(L_{\psi} f\right)(\cdot, a)-\left(L_{\varphi} f\right)(\cdot, a)\right\|_{m, p, \omega} \leq C^{1}|a|^{n / 2}\left\|\frac{\psi}{\sqrt[2 n]{C_{\psi}}}-\frac{\varphi}{\sqrt[2 n]{C_{\varphi}}}\right\|_{1}\|f\|_{m, p, \omega},
$$

where

$$
C^{1}=\max _{|\mu| \leq m}\left(C_{1, \mu}\right)^{1 / p}
$$

Similarly we obtain

$$
\left\|\left(L_{\varphi} f\right)(\cdot, a)-\left(L_{\varphi} g\right)(\cdot, a)\right\|_{m, p, \omega} \leq C^{1}|a|^{n / 2} \frac{\|\varphi\|_{1}}{\sqrt[2 n]{C_{\varphi}}}\|f-g\|_{m, p, \omega} .
$$

By the triangle inequality we get

$$
\begin{aligned}
& \left\|\left(L_{\psi} f\right)(\cdot, a)-\left(L_{\varphi} g\right)(\cdot, a)\right\|_{m, p, \omega} \\
& \quad \leq C^{1}|a|^{n / 2}\left(\left\|\frac{\psi}{\sqrt[2 n]{C_{\psi}}}-\frac{\varphi}{\sqrt[2 n]{C_{\varphi}}}\right\|\|f\|_{m, p, \omega}+\frac{\|\varphi\|_{1}}{\sqrt[2 n]{C_{\varphi}}}\|f-g\|_{m, p, \omega}\right) .
\end{aligned}
$$

\section{Symptotical behaviour for small dilation parameter}

From Theorem 2.2 the following proposition follows immediately.

Proposition 3.1. If $\psi$ is a basic wavelet and $f \in W_{\omega}^{m, p}\left(\mathbb{R}^{n}\right)$, then

$$
\left\|\left(L_{\psi} f\right)(\cdot, a)\right\|_{m, p, \omega}=O\left(|a|^{n / 2}\right) .
$$

Now consider the operator

$$
\left(\Lambda_{\psi} f\right)(b, a)=\left(\psi_{a} * f\right)(b)=\frac{1}{a^{n}} \int_{\mathbb{R}^{n}} f(t) \psi\left(\frac{b-t}{a}\right) d t
$$

where $\psi \in L^{1}\left(\mathbb{R}^{n}\right), f \in L^{p}\left(\mathbb{R}^{n}\right), 1 \leq p<\infty$, and

$$
\psi_{a}(x)=\frac{1}{a^{n}} \psi\left(\frac{x}{a}\right), \quad a \neq 0 .
$$

In the sequel, the following lemma is needed.

Lemma 3.2. Let $f \in W_{\omega}^{m, p}\left(\mathbb{R}^{n}\right), 1 \leq p<\infty, \psi \in L^{1}\left(\mathbb{R}^{n}\right) \cap L^{q}\left(\mathbb{R}^{n}\right)$, with

$$
\int_{\mathbb{R}^{n}} \psi(t) d t=1, \quad \frac{1}{p}+\frac{1}{q}=1 .
$$


140 The integral wavelet transform in weighted Sobolev spaces

Then

(i) $\left(\Lambda_{\psi} f\right)(\cdot, a) \rightarrow f(\cdot)$ in $W_{\omega}^{m, p}\left(\mathbb{R}^{n}\right)$ as $a \rightarrow 0^{+}$;

(ii) $\left[\partial^{\mu}\left(\Lambda_{\psi} f\right)\right](\cdot, a)=\left[\Lambda_{\psi}\left(\partial^{\mu} f\right)\right](\cdot, a)=a^{-|\mu|}\left(\Lambda_{\partial^{\mu} \psi} f\right)(\cdot, a)$ if $\partial^{\mu} \psi \in L^{1}\left(\mathbb{R}^{n}\right)$ $\cap L^{q}\left(\mathbb{R}^{n}\right),|\mu|<m, a>0$.

Proof. Since $\left(\Lambda_{\psi} f\right)(\cdot, a)=\left(\psi_{a} * f\right)(\cdot)$, and for each multi-index $\alpha, \sum_{i=1}^{n} \alpha_{i} \leq m$ such that $\partial^{\mu} f \in L^{p}\left(\mathbb{R}^{n}\right), \psi \in L^{q}\left(\mathbb{R}^{n}\right)$, we obtain

$$
\begin{gathered}
{\left[\partial^{\mu}\left(\psi_{a} * f\right)\right](\cdot)=\left(\psi_{a} * \partial^{\mu} f\right)(\cdot)} \\
\left\|\left(\Lambda_{\psi} f\right)(\cdot, a)-f(\cdot)\right\|_{m, p, \omega} \\
=\sum_{|\mu| \leq m}\left(\int_{\mathbb{R}^{n}} \omega_{\mu}(\cdot)\left|\partial^{\mu}\left[\left(\Lambda_{\psi} f\right)(\cdot, a)-f(\cdot)\right]\right|^{p} d(\cdot)\right)^{1 / p} \\
=\sum_{|\mu| \leq m}\left(\int_{\mathbb{R}^{n}} \omega_{\mu}(\cdot)\left|\Lambda_{\psi}\left(\partial^{\mu} f\right)(\cdot, a)-\left(\partial^{\mu} f\right)(\cdot)\right|^{p} d(\cdot)\right)^{1 / p} .
\end{gathered}
$$

Taking into account that $\partial^{\mu} f \in L^{p}\left(\mathbb{R}^{n}\right)$, it is easy to see that

$$
\begin{aligned}
& \int_{\mathbb{R}^{n}} \omega_{\mu}(\cdot)\left|\Lambda_{\psi}\left(\partial^{\mu} f\right)(\cdot, a)-\left(\partial^{\mu} f\right)(\cdot)\right|^{p} d(\cdot) \\
& \quad \leq\left\|\omega_{\mu}\right\|_{\infty} \int_{\mathbb{R}^{n}}\left|\Lambda\left(\partial^{\mu} f\right)(\cdot, a)-\left(\partial^{\mu} f\right)(\cdot)\right|^{p} d(\cdot) \longrightarrow 0 \quad \text { as } a \longrightarrow 0^{+} .
\end{aligned}
$$

Consequently, $\left(\Lambda_{\psi} f\right)(\cdot, a) \rightarrow f(\cdot)$ in $W_{\omega}^{m, p}\left(\mathbb{R}^{n}\right)$ as $a \rightarrow 0^{+}$, that is, (i) is proved.

To check (ii) take $f_{r} \in \mathscr{S}\left(\mathbb{R}^{n}\right), f_{r} \rightarrow f$ in $W_{\omega}^{m, p}\left(\mathbb{R}^{n}\right)$. It is obvious that in $W_{\omega}^{m-|\mu|, p}\left(\mathbb{R}^{n}\right)$

$$
\left[\partial^{\mu}\left(\Lambda_{\psi} f_{r}\right)\right](\cdot, a)=\left[\Lambda_{\psi}\left(\partial^{\mu} f_{r}\right)\right](\cdot, a)=a^{-|\mu|}\left(\Lambda_{\partial^{\mu} \psi} f_{r}\right)(\cdot, a) .
$$

By the continuity of the operators

$$
\begin{aligned}
& \Lambda_{\psi}: W_{\omega}^{m, p}\left(\mathbb{R}^{n}\right) \longrightarrow W_{\omega}^{m, p}\left(\mathbb{R}^{n}\right), \\
& \partial^{\mu}: W_{\omega}^{m, p}\left(\mathbb{R}^{n}\right) \longrightarrow W_{\omega}^{m-|\mu|, p}\left(\mathbb{R}^{n}\right),
\end{aligned}
$$

for $\psi \in L^{q}\left(\mathbb{R}^{n}\right),|\mu|<m$, letting $r \rightarrow \infty$ we get (ii).

Theorem 3.3. Let $f \in W_{\omega}^{m, p}\left(\mathbb{R}^{n}\right), 1 \leq p<\infty, \psi \in L^{1}\left(\mathbb{R}^{n}\right) \cap L^{q}\left(\mathbb{R}^{n}\right)$ such that

$$
\int_{\mathbb{R}^{n}} \psi(t) d t=1
$$

Moreover, assume that $\partial^{\mu} \psi$ is a basic wavelet for each multi-index $\mu,|\mu| \in$ $\{0,1,2, \ldots, m\}$, and $\partial^{\mu} \psi \in L^{q}\left(\mathbb{R}^{n}\right)$, with $1 / p+1 / q=1$. Suppose additionally that $f$ and $\psi$ are real-valued functions and $a>0$. Then

$$
\lim _{a \rightarrow 0^{+}}\left\|\frac{1}{a^{|\mu|+n / 2}}\left(L_{\partial^{\mu} \psi} f\right)(\cdot,-a)-\frac{1}{\sqrt[2 n]{C_{\partial^{\mu} \psi}}} \partial^{\mu} f(\cdot)\right\|_{m-|\mu|, p, \omega}=0 .
$$


Proof. Obviously

$$
\begin{aligned}
\left(L_{\partial^{\mu} \psi} f\right)(\cdot,-a) & =\frac{a^{n / 2}}{\sqrt[2 n]{C_{\partial^{\mu} \psi}}}\left[\left(\partial^{\mu} \psi\right)_{a} * f\right](\cdot) \\
& =\frac{a^{n / 2}}{\sqrt[2 n]{C_{\partial^{\mu} \psi}}}\left(\psi_{a} * \partial^{\mu} f\right)(\cdot)=\frac{a^{|\mu|+n / 2}}{\sqrt[2 n]{C_{\partial^{\mu} \psi}}}\left[\partial^{\mu}\left(\psi_{a} * f\right)\right](\cdot) .
\end{aligned}
$$

Under the assumptions of the theorem, the differentiation and integration can be interchanged, and furthermore by the continuity of the operator

$$
\partial^{\mu}: W_{\omega}^{m, p}\left(\mathbb{R}^{n}\right) \longrightarrow W_{\omega}^{m-|\mu|, p}\left(\mathbb{R}^{n}\right)
$$

for $|\mu|<m$, we get

$$
\begin{gathered}
\left\|\frac{1}{a^{|\mu|+n / 2}}\left(L_{\partial^{\mu} \psi} f\right)(\cdot,-a)-\frac{1}{\sqrt[2 n]{C_{\partial^{\mu} \psi}}}\left(\partial^{\mu} f\right)(\cdot)\right\|_{m-|\mu|, p, \omega} \\
\leq \frac{1}{\sqrt[2 n]{C_{\partial^{\mu} \psi}}}\left\|\left[\partial^{\mu}\left(\psi_{a} * f\right)\right](\cdot)-\left(\partial^{\mu} f\right)(\cdot)\right\|_{m-|\mu|, p, \omega} \\
\leq \frac{C}{\sqrt[2 n]{C_{\partial^{\mu} \psi}}}\left\|\left(\psi_{a} * f\right)(\cdot)-f(\cdot)\right\|_{m, p, \omega} \cdot
\end{gathered}
$$

Lemma 3.2 implies now that the last term in (3.13) tends to 0 as $a \rightarrow 0^{+}$.

\section{Acknowledgment}

This paper was supported by the National Fundamental Research Fund Grant for Natural Science, Vietnam.

\section{References}

[1] R. A. Adams, Sobolev Spaces, Pure and Applied Mathematics, vol. 65, Academic Press, New York, 1975.

[2] N. M. Chuong, N. M. Tri, and L. Q. Trung, Theory of Partial Differential Equations, Science and Technology Publishing House, Hanoi, 1995.

[3] N. M. Chuong and T. N. Tri, The integral wavelet transform in $L^{p}\left(\mathbf{R}^{n}\right), 1 \leq p \leq \infty$, Fract. Calc. Appl. Anal. 3 (2000), no. 2, 133-140.

[4] L. Hörmander, The Analysis of Linear Partial Differential Operators. I. Distribution Theory and Fourier Analysis, Fundamental Principles of Mathematical Sciences, vol. 256, Springer-Verlag, Berlin, 1983.

[5] Y. Meyer, Ondelettes et Opérateurs. I [Wavelets and Operators. I], Hermann, Paris, 1990.

[6] A. Rieder, The wavelet transform on Sobolev spaces and its approximation properties, Numer. Math. 58 (1991), no. 8, 875-894. 
142 The integral wavelet transform in weighted Sobolev spaces

Nguyen Minh Chuong: Institute of Mathematics, P.O. Box 631, Bo Ho, 10000 Hanoi, VieTnam

E-mail address: nmchuong@thevinh.ncst.ac.vn

Ta Ngoc Tri: University of Pedagogy Hanoi II, Me Linh, Vinh Phu, Vietnam 


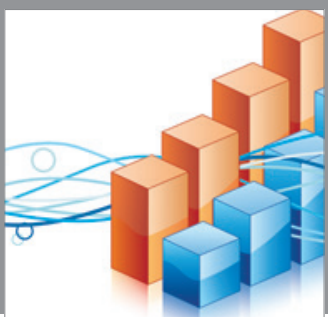

Advances in

Operations Research

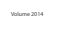

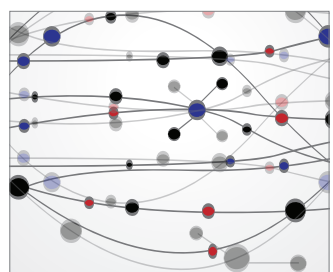

\section{The Scientific} World Journal
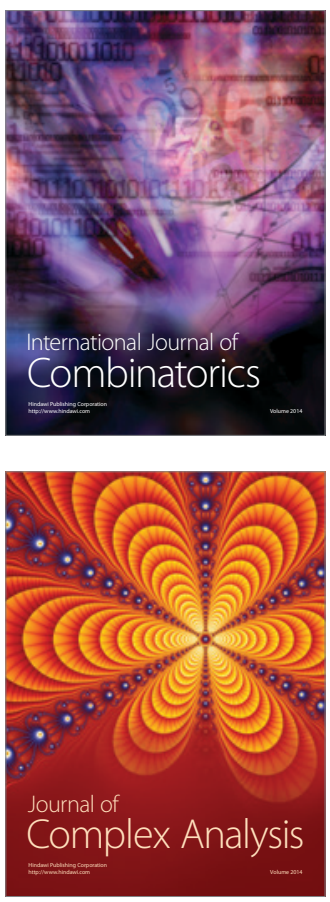

International Journal of

Mathematics and

Mathematical

Sciences
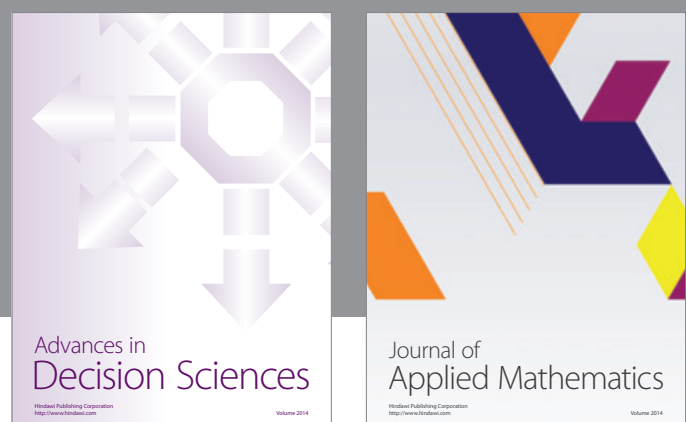

Journal of

Applied Mathematics
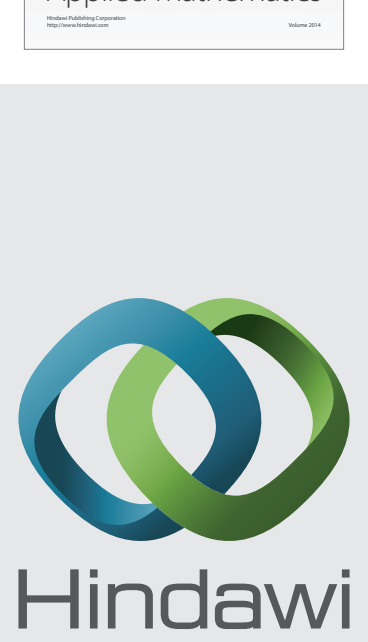

Submit your manuscripts at http://www.hindawi.com
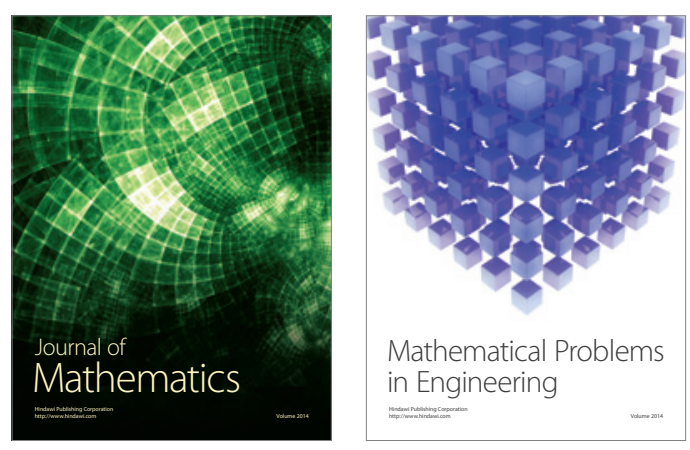

Mathematical Problems in Engineering
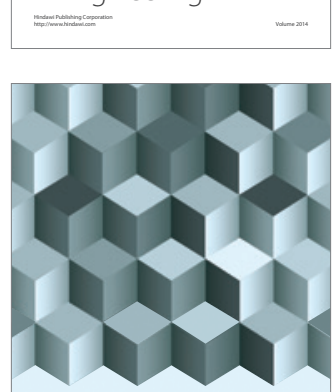

Journal of

Function Spaces
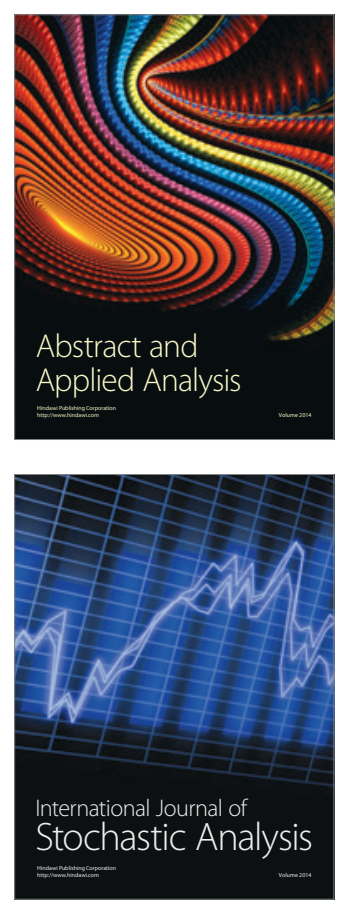

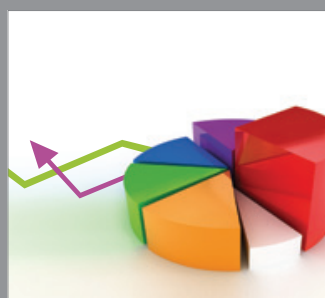

ournal of

Probability and Statistics

Promensencen
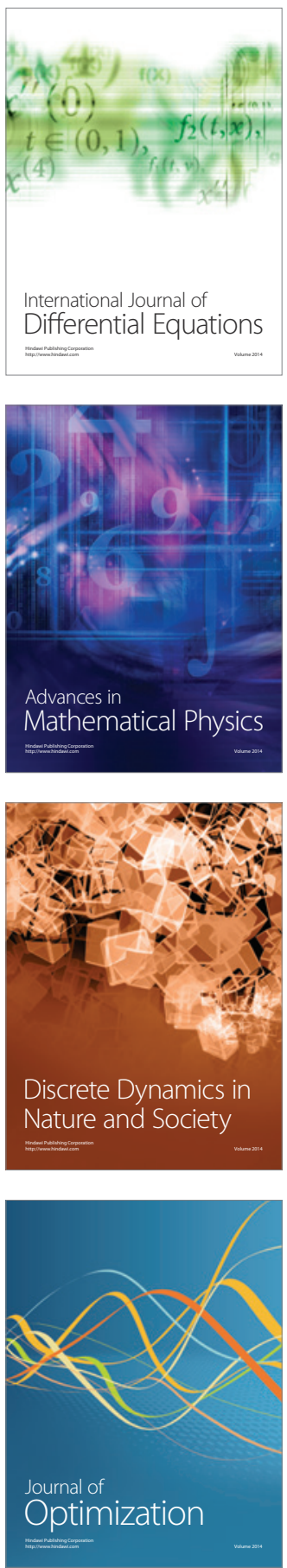